13.1

\title{
Роль кулоновского взаимодействия электронов адсорбата и субстрата: модель поверхностного димера
}

\author{
() С.Ю. Давыдов ${ }^{1}$, А.В. Зубов ${ }^{2}$, А.А. Лебедев ${ }^{1}$ \\ ${ }^{1}$ Физико-технический институт им. А.Ф. Иоффре РАН, Санкт-Петербург, Россия \\ ${ }^{2}$ Санкт-Петербургский национальный исследовательский университет информационных технологий, механики и оптики \\ (Университет ИТМО), Санкт-Петербург, Россия \\ E-mail: Sergei_Davydov@mail.ru
}

Поступило в Редакцию 27 мая 2019 г.

В окончательной редакции 27 мая 2019г.

Принято к публикации 7 июня 2019г.

Кулоновское взаимодействие в адсорбционной системе сведено к короткодействующему отталкиванию электронов адсорбированной частицы и атома подложки, образующих поверхностный димер. Показано, что учет такого взаимодействия приводит к увеличению перехода заряда между компонентами димера и уменьшению перехода заряда между димером и металлической подложкой, вызванного вариацией уровня Ферми адсорбционной системы.

Ключевые слова: кулоновское отталкивание, функция Грина, переход заряда, адсорбат, субстрат.

DOI: 10.21883/PJTF.2019.18.48232.17895

Интерес к роли кулоновского взаимодействия в задаче об адсорбции возник с момента появления первой работы [1], где использовался гамильтониан Андерсона [2], в рамках которого рассматривалось кулоновское отталкивание электронов с противоположными спинами на адатоме (внутриатомное кулоновское отталкивание). Результаты этой и подобных работ были обобщены на случай конечной концентрации адатомов вплоть до монослойного покрытия [3,4]. В дальнейшем было учтено также отталкивание электронов, принадлежащих соседним атомам адсорбированного слоя (межатомное кулоновское отталкивание) [4]. В настоящей работе мы рассмотрим кулоновское отталкивание электронов адсорбированной частицы и подложки и выясним, как учет такого взаимодействия влияет на переход заряда. Удобнее всего осуществить эту программу, воспользовавшись моделью поверхностного димера [5], которая строится следующим образом.

Рассмотрим свободный (не связанный с подложкой) димер, состоящий из частицы, изначально содержащей на орбитали с энергией $\varepsilon_{a}$ один электрон (дырку), и связанный с этой частицей поверхностный атом подложки, характеризуемый одноэлектронным уровнем с энергией $\varepsilon_{s}$. Соответствующий гамильтониан имеет вид

$$
H_{d i m}^{0}=\varepsilon_{s} \hat{n}_{s}+\varepsilon_{a} \hat{n}_{a}+G \hat{n}_{s} \hat{n}_{a}-t\left(s^{+} a+a^{+} s\right) .
$$

Здесь $G$ - межатомное кулоновское отталкивание, $\hat{n}_{s}=s^{+} s$ и $\hat{n}_{a}=a^{+} a-$ операторы чисел заполнения для атома подложки в состоянии $|s\rangle$ и частицы в состоянии $|a\rangle, s^{+}(s)$ и $a^{+}(a)-$ соответствующие операторы рождения (уничтожения), $t$ - энергия перехода электрона между частицей и поверхностным атомом подложки. В рамках расширенной теории Хартри-Фока [1-4] гамильтониан (1) можно преобразовать к виду

$$
H_{d i m}^{0}=w_{s} \hat{n}_{s}+w_{a} \hat{n}_{a}-t\left(s^{+} a+a^{+} s\right)-G n_{s} n_{a},
$$

где $w_{s(a)}=\varepsilon_{s(a)}+G n_{a(s)}, n_{s(a)}=\left\langle\hat{n}_{s(a)}\right\rangle,\langle\ldots\rangle$ означает усреднение по основному состоянию гамильтониана (1). Исходя из уравнения Дайсона [1-4] получим функции Грина, соответствующие гамильтониану (2). Они равны

$$
G_{a(s)}^{0}(\omega)=\frac{g_{a(s)}(\omega)}{1-g_{a}(\omega) g_{s}(\omega) t^{2}},
$$

где $g_{a(s)}^{-1}(\omega)=\omega-w_{a(s)}+i 0^{+}$. Локальные уровни димеpa, определяемые полюсами функций Грина (3), равны

$$
\omega_{ \pm}=\bar{w} \pm R / 2, \quad R=\sqrt{\Delta^{2}+4 t^{2}},
$$

где $\bar{w}=\left(w_{a}+w_{s}\right) / 2, \Delta=w_{a}-w_{s}$. Соответствующие плотности состояний $\rho_{a(s)}^{0}(\omega)=-\pi^{-1} \operatorname{Im} G_{a(s)}^{0}(\omega)$ для частицы и атома

$$
\begin{gathered}
\rho_{a(s)}^{0}(\omega)=D_{ \pm} \delta\left(\omega-\omega_{+}\right)+D_{ \pm} \delta\left(\omega-\omega_{-}\right), \\
D_{ \pm}=(1 \pm \Delta / R) / 2,
\end{gathered}
$$

где $\delta(\ldots)$ - дельта-функция Дирака. Отметим, что состояния $\omega_{-}$и $\omega_{+}$отвечают связывающим и антисвязывающим состояниям димера, а множители $D_{-}$и $D_{+}-$ вероятностям заполнения этих состояний.

Учтем теперь взаимодействие димера с подложкой. Исходя из выражения (5) в пределе слабой связи димера с подложкой, т.е. при $\Gamma(\omega) \ll t$, легко показать, что плотности состояний компонентов димера могут быть приближенно представлены в виде

$$
\begin{gathered}
\rho_{a(s)}(\omega)=D_{ \pm} \rho_{+}(\omega)+D_{ \pm} \rho_{-}(\omega), \\
\rho_{\operatorname{dim}}(\omega)=\rho_{+}(\omega)+\rho_{-}(\omega), \\
\rho_{ \pm}(\omega)=\frac{1}{\pi} \frac{\Gamma(\omega)}{\left(\omega-\omega_{ \pm}-\Lambda(\omega)\right)^{2}+\Gamma^{2}(\omega)},
\end{gathered}
$$

где функция уширения $\Gamma(\omega)=\pi V^{2} \rho_{s}(\omega)\left(\rho_{s}(\omega)-\right.$ плотность состояний подложки, $V$ - усредненный по зоне 


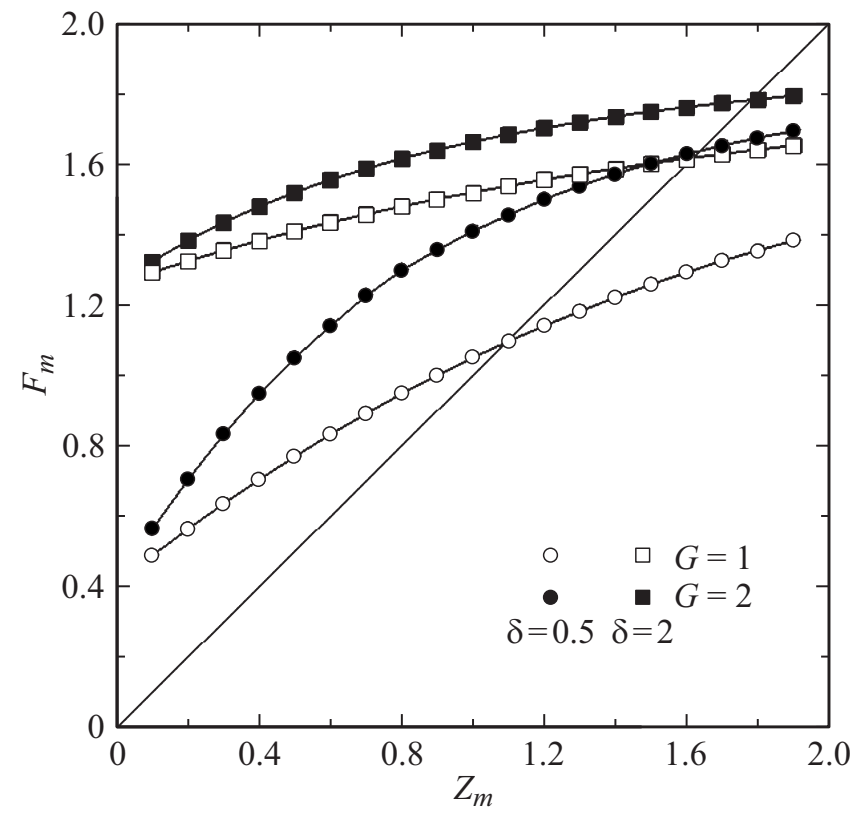

Схема решения уравнения (8). Точка пересечения прямой диагональной линии $Z_{m}$ с зависимостью $F_{m}\left(Z_{m}\right)$ определяет величину перехода заряда при $\varepsilon_{\mathrm{F}}=0$ и $\Gamma_{m}=0.25 t$. Светлые символы $-G=t$, темные $-G=2 t$; кружки $-\delta=0.5 t$, квадраты $-\delta=2 t$. Все энергетические параметры на рисунке приведены в единицах $t$.

Бриллюэна подложки матричный элемент взаимодействия частица-подложка), функция сдвига локальных уровней

$$
\Lambda(\omega)=V^{2} P \int_{-\infty}^{\infty} \rho_{s}\left(\omega^{\prime}\right)\left(\omega-\omega^{\prime}\right)^{-1} d \omega^{\prime}
$$

(P - символ главного значения) [5]. Конкретизируя природу подложки, задавая ее плотность состояний $\rho_{s}(\omega)$, будем предполагать, что изъятие из подложки одного атома не изменяет интегральной (средней по всей бесконечной поверхности) плотности состояний подложки.

В качестве конкретной адсорбционной системы рассмотрим адсорбцию на металле [6]. Для плотности состояний подложки примем приближение бесконечно широкой зоны модели Андерсона $[2,4]$, положив $\rho_{s}(\omega)=\rho_{m}=$ const, откуда получим $\Gamma(\omega)=\Gamma_{m}=\pi V^{2} \rho_{m}$ и $\Lambda(\omega)=\Lambda_{m}=0$. Тогда плотности состояний компонентов эпитаксиального димера (эпидимера) $\rho_{a(s)}^{m}(\omega)$ представляют собой сумму двух лоренцевых контуров $\rho_{ \pm}^{m}(\omega)=\Gamma_{m} / \pi\left[\left(\omega-\omega_{ \pm}\right)^{2}+\Gamma_{m}^{2}\right]$ с весовыми множителями $D_{ \pm}(4)$. При температуре $T=0$ соответствующие числа заполнения

$$
n_{ \pm}^{m}=2 \int_{-\infty}^{\varepsilon_{\mathrm{F}}} \rho_{ \pm}^{m}(\omega) d \omega
$$

(где $\varepsilon_{\mathrm{F}}-$ уровень Ферми системы) равны

$$
n_{a(s)}^{m}=D_{ \pm} n_{+}^{m}+D_{ \pm} n_{-}^{m}, \quad n_{d i m}^{0}=n_{+}^{m}+n_{-}^{m},
$$

где $n_{ \pm}^{m}=(2 / \pi) \operatorname{arccot}\left[\left(\omega_{ \pm}-\varepsilon_{\mathrm{F}}\right) / \Gamma_{m}\right]$. Здесь выражения (7) в отличие от случая $G=0$ [5] являются системой двух уравнений.

Полагая $\varepsilon_{s}+\varepsilon_{a}+2 G=0$, получим $\bar{w}=G\left(n_{s}+n_{a}-2\right)$. Если при этом уровень Ферми $\varepsilon_{\mathrm{F}}=0$, то переход заряда между димером и субстратом отсутствует, так что $n_{s}+n_{a}=2$ и $n_{ \pm}^{m}=1 \pm(2 / \pi) \arctan \left(R / 2 \Gamma_{m}\right)$, где $R$ дается выражением (4). Поскольку с ростом $G$ величина $R$ увеличивается, ясно, что учет межатомного кулоновского отталкивания усиливает переход заряда $n_{-}^{m}-n_{+}^{m}=2 v_{m}=(4 / \pi) \arctan \left(R / 2 \Gamma_{m}\right)$ между связывающим и антисвязывающим квазиуровнями. Величина перехода заряда между компонентами димера $Z_{m}=n_{s}^{m}-n_{a}^{m}$ определяется из уравнения

$$
Z_{m}=F_{m}, \quad F_{m}=2 \Delta v_{m} / R,
$$

где $\Delta=\delta+G Z_{m}$ и $\delta=\varepsilon_{a}-\varepsilon_{s}$. Подчеркнем, что знак $Z_{m}$ совпадает со знаком $\Delta$ и, следовательно, со знаком $\delta$. Если $\delta=0$, а $G \neq 0$, то вместо (8) получаем уравнение

$$
\left(\pi \Gamma_{m} / 2 G\right) x=\arctan x
$$

(где $x=G Z_{m} / 2 \Gamma_{m}$ ), которое имеет ненулевое решение при $\pi \Gamma_{m} / 2 G<1$. Отметим, что в случае $\varepsilon_{\mathrm{F}}=0$ димер представляет собой диполь с зарядами $\pm Z_{m} / 2$.

Схема решения уравнения (8) представлена на рисунке, из которого следует, что с ростом $G$ и $\delta$ переход заряда $Z_{m}$ растет. Отметим, что знак $Z_{m}$ совпадает со знаком $\delta$. Легко показать аналитически, что $d Z_{m} / d \delta=a Z_{m}$ и $d Z_{m} / d G=a Z_{m} /(1-a G)$, где $a=d Z_{m} / d \Delta \approx 8 t^{2} / R^{3}$ и учтено неравенство $\Gamma_{m} \ll R$. Значения $Z_{m}$ и производных $d Z_{m} / d \delta$ и $d Z_{m} / d G$ для случаев, изображенных на рисунке, представлены в таблице.

Пусть теперь $\varepsilon_{\mathrm{F}} \neq 0$, но $\left|\varepsilon_{\mathrm{F}}\right| \ll R$. Для числа заполнения димера $n_{d i m}=n_{+}^{m}+n_{-}^{m}$ (см. (7)) получим в линейном по $\varepsilon_{\mathrm{F}} / R$ приближении $n_{d i m} \approx 2\left(1+2 \varepsilon_{\mathrm{F}} \Gamma_{m} / \pi R^{2}\right)$ и $n_{-}^{m}-n_{+}^{m} \approx 2\left(1-2 \Gamma_{m} / \pi R\right)$. Отсюда следует, что с ростом $G$ и $\delta$ переход заряда между димером и металлом, вызванный вариацией уровня Ферми и равный $n_{d i m}-2$, уменьшается по модулю пропорционально $\left|\varepsilon_{\mathrm{F}}\right| / R$, тогда как переход заряда внутри димера, пропорциональный $n_{-}^{m}-n_{+}^{m}$, не меняется.

До сих пор мы рассматривали предел $\Gamma(\omega) \ll t$. Перейдем теперь к противоположному предельному случаю сильной связи димера с подложкой. С учетом взаимодействия с подложкой выражение (3) можно переписать, заменив функции Грина $g_{a(s)}^{-1}(\omega)=\omega-w_{a(s)}+i 0^{+}$ на $\bar{g}_{a(s)}^{-1}(\omega)=\omega-w_{a(s)}+i \Gamma_{m}$. Полагая $\Gamma_{m} \gg t$, в нулевом по $t^{2} / \Gamma_{m}^{2}$ приближении получим

$$
\begin{aligned}
& \bar{\rho}_{a(s)}(\omega)=\Gamma_{m} / \pi\left[\left(\omega-w_{a(s)}\right)^{2}+\Gamma_{m}^{2}\right], \\
& \bar{n}_{a(s)}=(2 / \pi) \operatorname{arccot}\left[\left(w_{a(s)}-\varepsilon_{\mathrm{F}}\right) / \Gamma_{m}\right] .
\end{aligned}
$$

Систему уравнений для $\bar{n}_{a(s)}$ можно свести к одному самосогласованному уравнению $G \bar{Z}_{m} \eta / \Gamma_{m}=\tan \left(\pi \bar{Z}_{m} / 2\right)$, имеющему ненулевое решение при $\pi \Gamma_{m} / 2 G<\eta \leqslant 1$, 
Зависимость перехода заряда $Z_{m}$ между компонентами димера от параметров $\delta$ и $G$ в режиме слабой связи димер-подложка (значения $\delta$ и $G$ приведены в единицах $t$, производные $d Z_{m} / d \delta, d Z_{m} / d G$ и $d Z_{m} / d \varepsilon_{\mathrm{F}}-$ в единицах $t^{-1}$ )

\begin{tabular}{c|c|c|c|c}
\hline Параметр & $\delta=0.5, G=1$ & $\delta=2, G=1$ & $\delta=0.5, G=2$ & $\delta=2, G=2$ \\
\hline$Z_{m}\left(\varepsilon_{\mathrm{F}}=0\right)$ & 1.63 & 1.70 & 1.10 & 1.62 \\
$d Z_{m} / d \delta\left(\varepsilon_{\mathrm{F}}=0\right)$ & 0.48 & 0.18 & 0.17 & 0.04 \\
$d Z_{m} / d G\left(\varepsilon_{\mathrm{F}}=0\right)$ & 0.91 & 0.21 & 0.21 & 0.04
\end{tabular}

где $\bar{Z}_{m}=\bar{n}_{s}-\bar{n}_{a}, \eta=\sin \left(\pi \bar{n}_{s} / 2\right) \sin \left(\pi \bar{n}_{a} / 2\right)$. Таким образом, в режиме сильной связи адсорбат-адсорбент, так же как и в режиме слабой связи, величина $\bar{Z}$ растет с ростом $G$. Полагая число заполнения димера $\bar{n}_{d i m}=\bar{n}_{s}+\bar{n}_{a}=2-\bar{v}(|\bar{v}| \ll 1)$, при $\left|\bar{Z}_{m}\right| \ll 1$ и $\left|\varepsilon_{\mathrm{F}}\right| / \Gamma_{m} \ll 1$ получим $\bar{v} \approx-4 \varepsilon_{\mathrm{F}} /\left(2 G+\pi \Gamma_{m}\right)$. Следовательно, с ростом $G$ величина перехода заряда, вызванного изменением уровня Ферми, понижается, как и в случае слабой связи. Далее с точностью до $t^{2} / \Gamma_{m}^{2}$ из (9) получим

$$
\rho_{a(s)}(\omega) \approx \bar{\rho}_{a(s)}(\omega)\left(1-t^{2} \frac{\Gamma_{m}^{2}-\Omega_{a(s)}\left(\Omega_{a(s)}+2 \Omega_{s(a)}\right)}{D(\omega)}\right),
$$

где $D(\omega)=\Omega_{a}^{2} \Omega_{s}^{2}+\Gamma_{m}^{2}\left(\Omega_{a}^{2}+\Omega_{s}^{2}\right)+\Gamma_{m}^{4}$ и $\Omega_{a(s)}=\omega-w_{a(s)}$. Заменяя в (10) энергию $\Omega_{a(s)}$ на $\bar{\Omega}_{a(s)}=\omega-\varepsilon_{a(s)}-G \bar{n}_{s(a)}$ и пренебрегая во втором слагаемом в круглых скобках членами $\sim \bar{v}, Z_{m}$, получим поправку к числу заполнения $\bar{n}_{a(s)}$ порядка $\varepsilon_{a(s)} \Gamma_{m} t^{2} /\left(\varepsilon_{a(s)}^{2}+\Gamma_{m}^{2}\right)^{2}$ [4]. Отсюда следует, что поправка к $\bar{Z}_{m}$ имеет третий порядок малости, так что ее учет не меняет качественных результатов, полученных на основании выражений (9).

Аналогичные оценки могут быть сделаны и для адсорбции на полупроводниковой и графеновой подложках (см. схемы расчетов, предложенные в [5]). Здесь, однако, мы ограничимся простейшем случаем адсорбции на металле.

В заключение скажем несколько слов об области применимости модели поверхностного димера. Представляется, что предложенная модель наиболее подходит к адсорбатам, которые могут образовывать с субстратом химические соединения, стабильные в свободном состоянии. В случае металлической подложки таковыми являются, например, атомы (молекулы) кислорода (металлические оксиды [7]) и иные газовые атомы и молекулы [8], а также элементы, образующие интерметаллиды и химические соединения на основе переходных металлов [9].

\section{Конфликт интересов}

Авторы заявляют, что у них нет конфликта интересов.
[3] Davison S.G., Sulston K.W. Green-function theory of chemisorption. Berlin: Springer, 2006. 211 p.

[4] Давыдов С.Ю. Теория адсорбции: метод модельных гамильтонианов. СПб.: Изд-во СПбГЭТУ „ЛЭТИ“, 2013. 235 с.; twirpx.com/file/1596114/

[5] Давыдов С.Ю., Зубов А.В., Лебедев А.А. // Письма в ЖТФ. 2019. T. 45. В. 9. C. $40-42$.

[6] Браун О.М., Медведев В.К. // УФН. 1989. Т. 157. В. 4. C. 631-666.

[7] Henrich V.E., Cox P.A. The surface science of metal oxides. Cambridge: Cambridge University Press, 1994. 464 p.

[8] Ramsier R.D., Yates J.T., Jr. // Surf. Sci. Rep. 1991. V. 12. N 6-8. P. 243-378.

[9] Вонсовский С.В., Изюмов Ю.А., Курмаев Э.З. Сверхпроводимость переходных металлов, их сплавов и соединений. М.: Наука, 1977. 384 c.

\section{Список литературы}

[1] Newns D.M. // Phys. Rev. 1969. V. 178. N 4. P. 1123-1135.

[2] Anderson P.W. // Phys. Rev. 1961. V. 124. N 1. P. 41-53. 